\title{
MERTON JUMP-DIFFUSION MODEL VERSUS THE BLACK AND SCHOLES APPROACH FOR THE LOG-RETURNS AND VOLATILITY SMILE FITTING
}

\author{
Nicola Gugole \\ Department of Computer Science \\ University of Verona \\ Strada le Grazie, 15-37134, Verona, ITALY
}

\begin{abstract}
In the present paper we perform a comparison between the standard Black and Scholes model and the Merton jump-diffusion one, from the point of view of the study of the leptokurtic feature of log-returns and also concerning the volatility smile fitting. Provided results are obtained by calibrating on market data and by mean of numerical simulations which clearly show how the jump-diffusion model outperforms the classical geometric Brownian motion approach.
\end{abstract}

AMS Subject Classification: 60H15, 60H35, 91B60, 91G20, 91G60

Key Words: Black and Scholes model, Merton model, stochastic differential equations, log-returns, volatility smile

\section{Introduction}

In the early 1970's the world of option pricing experienced a great contribution given by the work of Fischer Black and Myron Scholes. They developed a new mathematical model to treat certain financial quantities publishing related results in the article The Pricing of Options and Corporate Liabilities, see [1]. The latter work became soon a reference point in the financial scenario.

$\begin{array}{lr}\text { Received: } & \text { August 3, } 2016 \\ \text { Revised: } & \text { September 16, 2016 } \\ \text { Published: } & \text { September 30, 2016 }\end{array}$

(c) 2016 Academic Publications, Ltd. url: www.acadpubl.eu 
Nowadays, many traders still use the Black and Scholes (BS) model to price as well as to hedge various types of contingent claims. An important property of the BS model is that all the involved parameters are not influenced by the risk preferences of investors. In particular, the BS approach is based on the socalled risk-neutral pricing assumption which greatly simplifies the associated derivatives analysis.

In particular, in the classical BS-model, the volatility parameter, let us indicate it with $\sigma$, is assumed to be constant. Latter hypothesis cannot be considered realistic, as simple empirical analyses can easily show. In particular it is rather simple to show that the implied volatility of a financial asset is not constant but varies with time to maturity $T>0$, and with respect to the strike price $K$. Such a fact has started to become more and more evident since the general market crash in 1987. As a consequence the real values of the volatility parameter that can be observed in the market do not give rise to a flat shape as the BS-model forecasts. In fact, if we fix the strike price value and we look at the corresponding implied volatility section, e.g. with respect to a plain vanilla option, the typical figure that appears justifies the definition of the so-called smile/smirk effect. The latter because, especially for short maturities, the implied volatility sections assume a shape which resembles a smile or a smirk. Moreover, observing the log-returns time series of the major stock indexes, it is evident that the tails of the related distributions decay slowly to infinite and this means that the stock price is often characterized by big fluctuations, which happens at any time scale and are mainly due to unpredictable events with high financial impact, the so-called black swans, or rare events. In what follows, we recall the main reasons why the BS-model is not able to reproduce the aforementioned empirical facts which, in turn, are responsible for the observed leptokurtic feature of the log-returns, a key point to obtain realistic forecasts and simulations for the assets' prices.

As a consequence of the BS-model lack of description accuracy, new models have been developed to overcome issues of the type mentioned so far. This has been also produced approaches able to treat the increasingly complexity characterizing modern financial instruments. Between such alternatives to the BS analysis, we focus our attention on the proposal given in 1976 by Merton, see [13]. He allowed the underlying dynamics to have random jumps and also to reproduce more realistic tails behavior for related log-returns. The seminal approach suggested by Merton gave rise to the development of what are now known as jump-diffusion type models. In these models, the concept of sudden and unpredictable events is intrinsic because of the presence of a random noise of jump-type, a feature that cannot be taken into account by models based 
only on a Brownian type of noise, the latter being characterized by continuous trajectories.

Moreover, the introduction of (random) jump addends also brings benefits to what concerns a better reproduction of the aforementioned smile effect, a feature which plays a key role in the option pricing framework.

\section{The Financial Framework}

In order to introduce the financial framework we will work with, we consider a filtered probability space $\left(\Omega, \mathcal{F},\left\{\mathcal{F}_{t}\right\}_{0 \leq t \leq T}, \mathbb{P}\right)$, where $\Omega$ is the set of all the possible events, $\left\{\mathcal{F}_{t}\right\}_{0 \leq t \leq T}$ is a filtration which satisfies $\mathcal{F}_{T}=\mathcal{F}$, while $T>0$ is a real constant representing the maturity date of all our investments, and $\mathbb{P}$ represents the so-called real-world measure. Let us suppose that there are two assets in the market: a riskless asset and a stock whose related prices are respectively modeled by the following $\mathcal{F}_{t}$-adapted stochastic processes: $\left\{B_{t}\right\}_{0 \leq t \leq T}$, and $\left\{S_{t}\right\}_{0 \leq t \leq T}$. The riskless asset ensures a certain future return, specified by a riskless interest rate which models the money market account. On the other hand, to have a concrete example in hand, let us consider the stock to be the underlying of a European call option, namely the underlying of a contract that gives its holder the right, but not the obligation, to buy one unit of the stock for a predetermined strike price $K>0$, at maturity time $T$. The payoff of such a call option is defined by

$$
\left(S_{T}-K\right)^{+} \doteq \begin{cases}S_{T}-K, & \text { if } S_{T}>K \\ 0, & \text { if } S_{T} \leq K\end{cases}
$$

In the first case the holder will exercise the option and make a profit equal to $S_{T}-K$, buying the stock for $K$ and selling it immediately at the market price $S_{T}$. In the second case the option is simply not exercised. In the following we make various assumptions concerning the financial market characteristics, mainly following [1] and [13]. In particular we assume that

- the riskless interest rate $r>0$ is known and constant through time. It models the evolution of the riskless asset price which satisfies the following ODE

$$
\left\{\begin{array}{l}
\left.B\right|_{t=0}=B_{0}>0 \\
d B_{t}=r B_{t} d t, 0<t \leq T
\end{array} \quad \Rightarrow B_{t}=B_{0} e^{r t}, \quad 0 \leq t \leq T\right.
$$


- the stock does not pay dividends: in particular if, as an example, the stock is related to some company ACME, then the latter means that the shareholder does not receive any distribution of the profit from the ACME company;

- Frictionless markets: there are no transaction costs nor taxes in buying or selling the stock or the option. Trading takes place continuously in time. Borrowing and short selling are allowed without restrictions. The borrowing and lending rates are equal to $r$;

- there are no riskless arbitrage opportunities. Shortly, it means that there is no way of trading on the stock so that an agent has probability equal to one to gain a strictly positive quantity of money.

We will consider two different ways to model the asset dynamics, each of which leads to different pricing formula for the same European call option.

\section{a) Black and Scholes model}

The stock price evolves according to the following stochastic differential equation

$$
\left\{\begin{array}{l}
\left.S\right|_{t=0}=S_{0}>0, \\
d S_{t}=\mu S_{t} d t+\sigma S_{t} d W_{t}, \quad 0<t \leq T,
\end{array}\right.
$$

$\left\{W_{t}\right\}_{0 \leq t \leq T}$ being a Brownian motion under the measure $\mathbb{P}$. In this particular context $\mu$ represents the annualized expected rate of return on the asset, while $\sigma$ is the annualized volatility of the asset. It is well known that this equation has a unique strong solution of the form

$$
S_{t}=S_{0} \exp \left\{\sigma W_{t}+\left(\mu-\frac{1}{2} \sigma^{2}\right) t\right\}, 0 \leq t \leq T,
$$

which is nothing but the definition of the geometric Brownian motion $(\mathrm{gBm})$. We refer to [16] and [17] for further details about Brownian motion, diffusion processes and related stochastic calculus. In particular, we recall that the distribution at time $t$ of the $\mathrm{gBm}$ is log-normal, indeed, for $0 \leq t \leq T$, we have

$$
\begin{aligned}
\log S_{t} & =\log S_{0}+\sigma W_{t}+\left(\mu-\frac{1}{2} \sigma^{2}\right) t \\
& \sim \mathcal{N}\left(\log S_{0}+\left(\mu-\frac{1}{2} \sigma^{2}\right) t, \sigma^{2} t\right),
\end{aligned}
$$

where $\mathcal{N}$ denotes the normal distribution, so that

$$
\log \left(\frac{S_{t}}{S_{0}}\right)=\sigma W_{t}+\left(\mu-\frac{1}{2} \sigma^{2}\right) t
$$




$$
\sim \mathcal{N}\left(\left(\mu-\frac{1}{2} \sigma^{2}\right) t, \sigma^{2} t\right)
$$

which implies that the log-returns are normally distributed.

In such a framework is well known that the market model is complete, and then the existence and uniqueness of the martingale measure $\mathbb{Q}$ is guaranteed, so that it is possible to perfectly hedge a short position on the derivative security, see, e.g., [14]. The associated asset's martingale dynamic reads as follow

$$
\left\{\begin{array}{l}
\left.S\right|_{t=0}=S_{0}>0, \\
d S_{t}=r S_{t} d t+\sigma S_{t} d W_{t}^{\mathbb{Q}}, \quad 0<t \leq T,
\end{array}\right.
$$

where

$$
W_{t}^{\mathbb{Q}} \doteq W_{t}+\frac{\mu-r}{\sigma} t, 0 \leq t \leq T
$$

is a $\mathbb{Q}$-Bwownian motion, as assured by the Girsanov Theorem. Finally we recall that the risk-neutral pricing formula, which express the European call option price at time $t$ with underlying price $x$, is given by

$$
C(t, x)=e^{-r \tau} \tilde{\mathbb{E}}\left[\left(S_{T}-K\right)^{+} \mid S_{t}=x\right], \tau=T-t,
$$

where $\tilde{\mathbb{E}}$ denotes the expectation under $\mathbb{Q}$. Latter equation leads to the celebrated Black and Scholes formula, see, e.g., [14]. In particular, indicating with $C_{B S}$ the call price under the Black and Scholes approach, we have

$$
C_{B S}(\tau, x)=x \Phi\left(d_{+}(\tau, x)\right)-e^{-r \tau} K \Phi\left(d_{-}(\tau, x)\right),
$$

where $\Phi$ is the cumulative distribution function for the standard normal random variable, namely

$$
\Phi(z) \doteq \frac{1}{\sqrt{2 \pi}} \int_{-\infty}^{z} e^{-\frac{y^{2}}{2}} d y
$$

and

$$
d_{ \pm}(\tau, x) \doteq \frac{1}{\sigma \sqrt{\tau}}\left\{\log \left(\frac{x}{K}\right)+\left(r \pm \frac{\sigma^{2}}{2}\right) \tau\right\} .
$$

In order to estimate the volatility $\sigma$, a possible approach consists in computing the related implied volatility value. Given an observed call option price $C^{*}$ for a contract with strike price $K$ and expiration date $T$, the implied volatility $\Sigma>0$ is defined as the value that the volatility should have, if we take into account the BS formula (4), in order to match the associated BS price, namely

$$
C_{B S}(\tau, x ; \Sigma)=C^{*}
$$




\section{b) Merton model}

Let us introduce in what follows the Merton approach, starting by exploiting the following SDE to model del stock behaviour

$$
\left\{\begin{array}{l}
\left.S\right|_{t=0}=S_{0}>0, \\
d S_{t}=(\mu-\lambda k) S_{t} d t+\sigma S_{t} d W_{t}+S_{t-} d Q_{t}, \quad 0<t \leq T,
\end{array}\right.
$$

where $\left\{W_{t}\right\}_{0 \leq t \leq T}$ is a Brownian motion, and $\left\{Q_{t}\right\}_{0 \leq t \leq T}$ is a compound Poisson process of the form

$$
\begin{gathered}
Q_{t} \doteq \sum_{i=1}^{N_{t}}\left(Y_{i}-1\right), \\
Y_{i}=\frac{S_{T_{i}}}{S_{T_{i-}}}>0, i \in \mathbb{N},
\end{gathered}
$$

being the price ratio associated with the $i$-th jump along the path of the stock price, happened at random time $T_{i}>0$. We assume that the random variables $\left\{Y_{i}\right\}_{i \in \mathbb{N}}$ are i.i.d. and that they are also independent of both $W_{t}$ and $N_{t}$, the latter representing a Poisson process with intensity $\lambda>0$. Moreover we assume

$$
V_{i} \doteq \log Y_{i} \sim \mathcal{N}\left(m, \delta^{2}\right),
$$

with probability density

$$
f_{V}(y)=\frac{1}{\delta \sqrt{2 \pi}} e^{-\frac{(y-m)^{2}}{2 \delta^{2}}}, y \in \mathbb{R},
$$

as in the original specification of the model, see [13]. It follows that

$$
\begin{gathered}
\mathbb{E}\left[Q_{t}\right]=\lambda k t, 0 \leq t \leq T, \\
k \doteq \mathbb{E}\left[Y_{i}-1\right]=e^{m+\frac{\delta^{2}}{2}}-1,
\end{gathered}
$$

so that in equation (5) we have the compensated version of $Q_{t}$, which, in this form, turns to be a martingale. As in the BS-model case, we can count on an explicit solution also for the Merton model. In particular, we have

$$
S_{t}=S_{0} \exp \left\{\sigma W_{t}+\left(\mu-\lambda k-\frac{1}{2} \sigma^{2}\right) t\right\} \prod_{i=1}^{N_{t}} Y_{i}, 0 \leq t \leq T .
$$

If we consider the log-return for the Merton model, conditional on the event $\left\{N_{t}=j\right\}$, we can write 


$$
\begin{aligned}
\log \left(\frac{S_{t}}{S_{0}}\right)=\sigma W_{t}+(\mu-\lambda k & \left.-\frac{1}{2} \sigma^{2}\right) t+\sum_{i=1}^{j} V_{i} \\
& \sim \mathcal{N}\left(\left(\mu-\lambda k-\frac{1}{2} \sigma^{2}\right) t+j m, \sigma^{2} t+j \delta^{2}\right),
\end{aligned}
$$

which allows us to obtain its probability density as a quickly converging series. Indeed, for $A \subseteq \mathbb{R}$, we have

$$
\mathbb{P}\left(\log \left(\frac{S_{t}}{S_{0}}\right) \in A\right)=\sum_{j=0}^{\infty} \mathbb{P}\left(\log \left(\frac{S_{t}}{S_{0}}\right) \in A \mid N_{t}=j\right) \mathbb{P}\left(N_{t}=j\right),
$$

hence the related probability density at time $t$ reads as follow

$$
\psi_{t}(y)=e^{-\lambda t} \sum_{j=0}^{\infty} \frac{(\lambda t)^{j} \exp \left\{-\frac{\left(y-\left(\mu-\lambda k-\frac{1}{2} \sigma^{2}\right) t-j m\right)^{2}}{2\left(\sigma^{2} t+j \delta^{2}\right)}\right\}}{j ! \sqrt{2 \pi\left(\sigma^{2} t+j \delta^{2}\right)}}, y \in \mathbb{R},
$$

hence it is expressed by a weighted sum of normal densities.

Before describing other properties of the Merton model, we would like to recall that such a jump-diffusion model, contrary to the BS one, is not complete. In particular, there are many possible choices to define a martingale measure, namely a measure $\mathbb{Q} \sim \mathbb{P}$ such that the discounted price $e^{-r t} S_{t}$ is a martingale. In fact, by a direct computation, we easily get

$$
\begin{array}{r}
d\left(e^{-r t} S_{t}\right)=e^{-r t}(\mu-r) S_{t} d t+e^{-r t} \sigma S_{t} d W_{t}+e^{-r t} S_{t-}\left(d Q_{t}-\lambda k d t\right) \\
=e^{-r t}(\mu-r-\lambda k) S_{t} d t+e^{-r t} \sigma S_{t}\left(d W_{t}^{\mathbb{Q}}-\theta d t\right)+e^{-r t} S_{t-} d Q_{t} \\
=e^{-r t}\left(\mu-r-\lambda k-\sigma \theta+\lambda^{\mathbb{Q}} k^{\mathbb{Q}}\right) S_{t} d t \\
\quad+e^{-r t} \sigma S_{t} d W_{t}^{\mathbb{Q}}+e^{-r t} S_{t-} d\left(Q_{t}-\lambda^{\mathbb{Q}} k^{\mathbb{Q}} t\right) .
\end{array}
$$

and imposing that such a differential is equal to zero, we obtain the following market price of risk equation

$$
\mu-r-\lambda k-\sigma \theta+\lambda^{\mathbb{Q}} k^{\mathbb{Q}}=0,
$$

where $\theta$ is such that

$$
W_{t}^{\mathbb{Q}} \doteq W_{t}+\theta t, 0 \leq t \leq T
$$

is a $\mathbb{Q}$-Bwownian motion, thanks to the Girsanov Theorem, while $\lambda^{\mathbb{Q}}>0$ is the new arrival rate and $k^{\mathbb{Q}} \doteq \tilde{\mathbb{E}}\left[Y_{i}-1\right]$. Merton proposed the following choice for the change of measure:

$$
\lambda^{\mathbb{Q}}=\lambda
$$




$$
\begin{aligned}
f_{V}^{\mathbb{Q}}(y) & =f_{V}(y) \Rightarrow k^{\mathbb{Q}}=k, \\
& \Rightarrow \theta=\frac{\mu-r}{\sigma} .
\end{aligned}
$$

Essentially he left the jump part unchanged, justifying such an hypotesis with the assumption that the jump risk is diversifiable and no risk premium is attached to it. In other words the latter means that the risk-neutral properties of the jump component of $S_{t}$ are supposed to be the same as its statistical properties. The dynamics of the stock price under $\mathbb{Q}$ are then

$$
S_{t}=S_{0} \exp \left\{\sigma W_{t}^{\mathbb{Q}}+\left(r-\lambda k-\frac{1}{2} \sigma^{2}\right) t\right\} \prod_{i=1}^{N_{t}} Y_{i}, 0 \leq t \leq T
$$

In the special case of jumps of Gaussian type length, it is possible to write, see $[13,8]$, the European call option price as follows

$$
\begin{aligned}
C_{M}(\tau, x) & =\sum_{j=0}^{\infty} e^{-\lambda \tau} \frac{(\lambda \tau)^{j}}{j !} \tilde{\mathbb{E}}\left[e^{-r \tau}\left(S_{T}-K\right)^{+} \mid S_{t}=x\right] \\
& =\sum_{j=0}^{\infty} e^{-\lambda \tau} \frac{(\lambda \tau)^{j}}{j !} C_{B S}\left(\tau, x_{j} ; \sigma_{j}\right)
\end{aligned}
$$

where

$$
\sigma_{j}^{2}=\sigma^{2}+\frac{j \delta^{2}}{\tau}, x_{j}=x \exp \left\{j m+\frac{j \delta^{2}}{2}-\lambda \tau e^{m+\frac{\delta^{2}}{2}}+\lambda \tau\right\} .
$$

If $\lambda=0$ then $C_{M}(\tau, x)=C_{B S}(\tau, x)$, indeed all the terms appearing in the sum are equal to 0 , except for $j=0$, when $x_{0}=x$ and $\sigma_{0}=\sigma$.

\section{The Comparison}

In what follows we show that the Merton model outperforms the BS-model, particularly to what concerns the following stylized empirical facts:

(1) the heavy tails which characterize the frequency distribution of log-returns;

(2) the volatility smile fitting in option pricing.

Concerning point (1), we exploit the approach provided in [10], first recalling the discretized form of the log-return SDE for both the BS and the Merton 
model. For the BS-model, if we deal with an interval of amplitude $\Delta t$, we can write

$$
\Delta \log S_{t}=\mu_{B S} \Delta t+\sigma_{B S} \Delta W_{t}
$$

where

$$
\Delta W_{t} \sim \mathcal{N}(0, \Delta t)
$$

while for the Merton model the related SDE reads as follow

$$
\Delta \log S_{t}=\mu_{M} \Delta t+\sigma_{M} \Delta W_{t}+V \Delta N_{t},
$$

where

$$
V \sim \mathcal{N}\left(m, \delta^{2}\right), \Delta N_{t} \sim \operatorname{Po}(\lambda \Delta t)
$$

All the quantities are assumed to be independent one from another and we write $E^{(a)}$, resp. $M_{i}^{(a)}$, resp. $s^{(a)}$, resp. $k^{(a)}$, to refer to the mean, resp. the $i$ th central moment, resp. the skewness, resp. the kurtosis for the model, or the time series, indicated by $a$.

Theorem 1. Exploiting eq. (14), we have

$$
\begin{gathered}
E^{(B S)}=\mu_{B S} \Delta t, M_{2}^{(B S)}=\sigma_{B S}^{2} \Delta t, \\
M_{3}^{(B S)}=0, M_{4}^{(B S)}=3 \sigma_{B S}^{4} \Delta t^{2}, \\
\Rightarrow s^{(B S)}=\frac{M_{3}^{(B S)}}{\left(M_{2}^{(B S)}\right)^{\frac{3}{2}}}=0, k^{(B S)}=\frac{M_{4}^{(B S)}}{\left(M_{2}^{(B S)}\right)^{2}}=3,
\end{gathered}
$$

while eq. (15) gives

$$
\begin{gathered}
E^{(M)}=\mu_{M} \Delta t+m \lambda \Delta t, \\
M_{2}^{(M)}=\sigma_{M}^{2} \Delta t+\left(\delta^{2}(1+\lambda \Delta t)+m^{2}\right) \lambda \Delta t, \\
M_{3}^{(M)}=m\left(3 \delta^{2}+m^{2}\right) \lambda \Delta t+6 m \delta^{2}(\lambda \Delta t)^{2}, \\
M_{4}^{(M)}=3\left(\sigma_{M}^{2} \Delta t\right)^{2}+\left(m^{4}+3 \delta^{4}+6 m^{2} \delta^{2}\right) \lambda \Delta t \\
+\left(3 m^{4}+21 \delta^{4}+30 m^{2} \delta^{2}\right)(\lambda \Delta t)^{2}+6 \sigma_{M}^{2} \Delta t\left(\delta^{2}+m^{2}\right) \lambda \Delta t \\
+\left(6 m^{2} \delta^{2}+18 \delta^{4}\right)(\lambda \Delta t)^{3}+6 \sigma_{M}^{2} \delta^{2} \Delta t(\lambda \Delta t)^{2}+3 \delta^{4}(\lambda \Delta t)^{4},
\end{gathered}
$$

from which $s^{(M)}$ and $k^{(M)}$ can be computed. Let us point out that in [10], the first term in (22) is substituted by $3\left(\sigma_{M} \Delta t\right)^{2}$. Nevertheless such a substitution turns out to be an error, as one can verify doing all the involved computations, in fact the term has the same form of the one derived for the fourth central moment in (17). 
Proof. The quantities in eq. (16) can be derived immediately applying the properties of the Brownian motion. To what concerns the central moments in eq. (17), we recall that

$$
M_{i}^{(B S)}=\mathbb{E}\left[\left(\mu_{B S} \Delta t+\sigma_{B S} \Delta W_{t}-\mu_{B S} \Delta t\right)^{i}\right]=\sigma_{B S}^{i} \mathbb{E}\left[\Delta W_{t}^{i}\right],
$$

and, since $\mathbb{E}\left[\Delta W_{t}^{i}\right]$ can be computed using the characteristic function of the normal random variable $\Delta W_{t}$, i.e.

$$
\phi_{\Delta W_{t}}(y)=e^{-y^{2} \frac{\Delta t}{2}}
$$

we have

$$
\mathbb{E}\left[\Delta W_{t}^{3}\right]=0, \mathbb{E}\left[\Delta W_{t}^{4}\right]=3 \Delta t^{2} .
$$

so that both the skewness and the kurtosis term appearing in eq. (18), easily follow. Switching to the Merton model, the mean term in eq. (19) can be obtained recalling that $\Delta N_{t} \sim \operatorname{Po}(\lambda \Delta t)$, while the central moments in eq. (20), (21) and eq. (22) are derived applying the following formula

$$
\begin{aligned}
M_{i}^{(M)}=\mathbb{E}\left[\left(\mu_{M} \Delta t+\sigma_{M} \Delta W_{t}+V \Delta\right.\right. & \left.\left.N_{t}-\mu_{M} \Delta t-m \lambda \Delta t\right)^{i}\right] \\
& =\mathbb{E}\left[\left(\sigma_{M} \Delta W_{t}+V \Delta N_{t}-m \lambda \Delta t\right)^{i}\right] .
\end{aligned}
$$

We omit the detailed computation, term by term, related to the equation (23), however it is useful to remember that we have assumed that every variable is independent of the others. Moreover, the moments of $V$ and $\Delta N_{t}$ are derived using the related characteristic functions,

$$
\phi_{V}(y)=e^{i m y-\frac{\delta^{2} y^{2}}{2}}, \phi_{\Delta N_{t}}(y)=e^{\lambda \Delta t\left(e^{i y}-1\right)},
$$

from which

$$
\begin{gathered}
\mathbb{E} V^{2}=m^{2}+\delta^{2}, \\
\mathbb{E} V^{3}=3\left(m^{2}+\delta^{2}\right) m-2 m^{3}, \\
\mathbb{E} V^{4}=3 \delta^{4}+6 m^{2}\left(m^{2}+\delta^{2}\right)-5 m^{4} .
\end{gathered}
$$

and

$$
\begin{gathered}
\mathbb{E} \Delta N_{t}^{2}=\lambda \Delta t+\lambda^{2} \Delta t^{2}, \\
\mathbb{E} \Delta N_{t}^{3}=\lambda \Delta t+3 \lambda^{2} \Delta t^{2}+\lambda^{3} \Delta t^{3}, \\
\mathbb{E} \Delta N_{t}^{4}=\lambda \Delta t+7 \lambda^{2} \Delta t^{2}+6 \lambda^{3} \Delta t^{3}+\lambda^{4} \Delta t^{4} .
\end{gathered}
$$


Using the aforementioned properties for the log-return processes, we focus our attention on empirical data. We consider the daily log-returns of the Standard \& Poor's (S\&P) 500 Index in the period from $03 \cdot 01 \cdot 2005$ to $10 \cdot 06 \cdot 2015$, taking $\Delta t=1 / 252 \simeq 0.004$, where the denominator 252 has been chosen following the convention that there are 252 trading days in a year. The conjecture is that this big mutual fund should not depend on the behavior of particular stocks and so the study should be more accurate. There is a total of 2621 daily closings and we have to deal with $n=2620$ log-returns. Moreover, from the S\&P 500 data it is possible to extract the following informations

$$
\begin{gathered}
E^{(S \& P)} \simeq 0.00021, M_{2}^{(S \& P)} \simeq 0.00016, \\
s^{(S \& P)} \simeq-0.33250<0, k^{(S \& P)} \simeq 14.201>3 .
\end{gathered}
$$

The goal is to find the vectors of parameters $\left(\mu_{B S}, \sigma_{B S}\right)$ for the BS-model, resp. $\left(\mu_{M}, \sigma_{M}, \lambda, m, \delta\right)$ for the Merton model. Then, we exploit such vectors to obtain a simulation of the log-returns as close as possible to the related time series. For the BS-model we impose

$$
E^{(B S)}=E^{(S \& P)}, M_{2}^{(B S)}=M_{2}^{(S \& P)},
$$

so that

$$
\mu_{B S}=\frac{E^{(S \& P)}}{\Delta t} \simeq 0.052645, \sigma_{B S}=\sqrt{\frac{M_{2}^{(S \& P)}}{\Delta t}} \simeq 0.20180
$$

recalling that a normal distribution is completely determined by its mean and variance. In the Merton model there are 5 parameters to estimate, hence, particularly in the practitioners' arena, it is standard to reduce this set assuming that

$$
E^{(M)}=E^{(S \& P)}, M_{2}^{(M)}=M_{2}^{(S \& P)},
$$

which implies

$$
\begin{gathered}
\mu_{M}=\frac{\mathrm{E}^{(S \& P)}-m \lambda \Delta t}{\Delta t}, \\
\sigma_{M}=\sqrt{\frac{M_{2}^{(S \& P)}-\left(\delta^{2}(1+\lambda \Delta t)+m^{2}\right) \lambda \Delta t}{\Delta t}},
\end{gathered}
$$

namely, the diffusion parameters are expressed as function of the jump ones, and we are left with the estimate of the 3 -dimensional vector $\eta \doteq(\lambda, m, \delta)$. To address the latter we use the Multinomial Maximum Likelihood approach, which can be summarized as follows: 
1. sort empirical data into $\tilde{n}<n$ bins, in order to get a computationally tractable problem. Then, for each of these bins, extract the sample frequency $f_{i}^{(S \& P)}, i=1, \ldots, \tilde{n}$;

2. construct the (theoretical) jump-diffusion frequency function

$$
f_{i}^{(M)}(\eta) \doteq n \int_{B_{i}} \psi_{\Delta t}(y ; \eta) d y, \quad i=1, \ldots, \tilde{n},
$$

where $B_{i}$ is the $i$ th bin and $\psi_{\Delta t}(y ; \eta)$ is the log-return probability density already seen in eq. (9);

3. minimize the objective function

$$
l(\eta) \doteq-\sum_{i=1}^{\tilde{n}} f_{i}^{(S \& P)} \log \left(f_{i}^{(M)}(\eta)\right),
$$

a task that can be completed also using built in routines which are present in various mathematical software, by starting with an initial point $\eta_{0}$. We would like to underline that even if the latter numerical approach is rather simple to be implemented, better results can be achieved using the so called regime switching analysis as, e.g., has been made in $[6,7]$, see also [11].

Following the aforementioned algorithm to treat the data at our disposal, we obtain:

$$
\begin{gathered}
\lambda \simeq 68.150, m \simeq-0.0025643, \delta \simeq 0.018482 \\
\Rightarrow \mu_{M} \simeq 0.22740, \sigma_{M} \simeq 0.10343,
\end{gathered}
$$

and exploiting equations (21) and (22), we have

$$
s^{(M)} \simeq-0.53531, k^{(M)} \simeq 13.859 .
$$

We can easily note that the skewness is bigger in absolute value than the one obtained using the real S\&P 500 data, but, unlike in the simple diffusion model with $s^{(B S)}=0$, the Merton approach tents to capture a clear absence of symmetry with the same sign. Moreover the kurtosis is well replicated, which is the main purpose of our analysis, while the BS-model provides poor results. It follows that the log-normal jump diffusion model represents a substantial and concrete improvement when compared to the classical diffusion model, particularly from the point of view of the log-returns simulation. Latter conclusions 


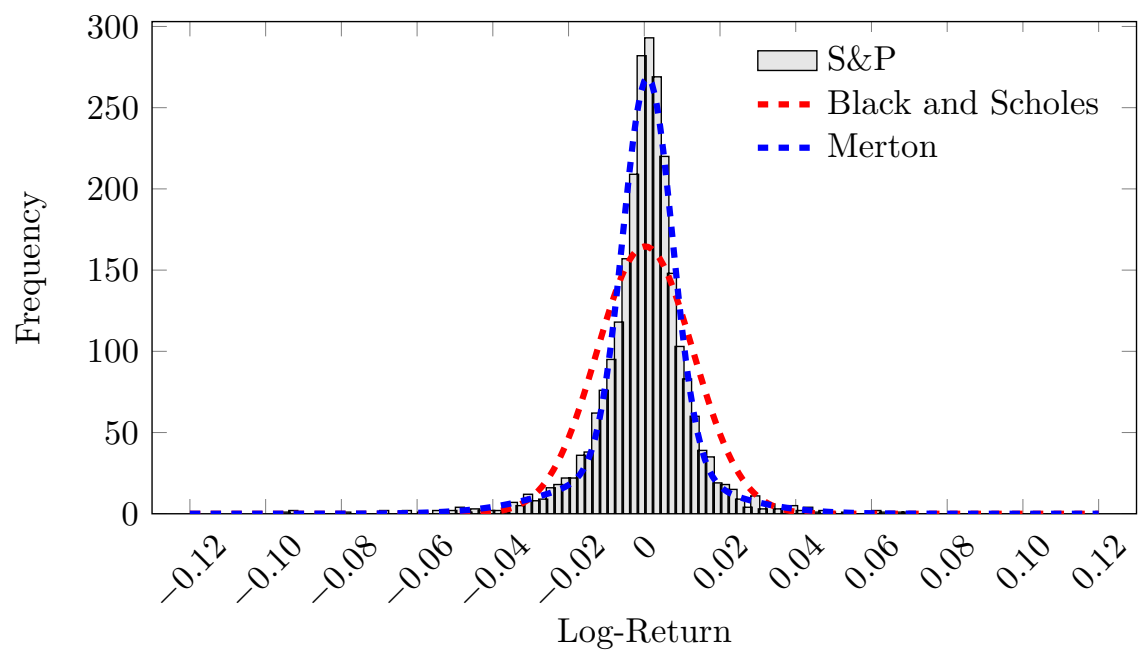

Figure 1: The empirical frequency distribution of S\&P 500 Index against the theoretical ones. For the Merton model the function in eq. (24) is computed for each bin. The construction for the BS-model is analogous. The code has been developed following $[8,10]$, then tested with data taken from [15].

are well reproduced in Fig. 1 which clearly represents the difference between the two models: the jump parameters $(\lambda, m, \delta)$ added to the set of diffusion parameters $\left(\mu_{M}, \sigma_{M}\right)$ allow to reproduce the leptokurtic feature of the log-returns.

In what follows, we compare the two models with respect to volatility smile fitting. We recall that if the BS-model were correct, then the implied volatility surface $\Sigma \doteq \Sigma(T, K)$ would be flat, namely it would remain constant for every strike price $K$ and for every maturity $T$. Unfortunately latter hypothesis is almost always not verified by real data. In particular, if we look at the (real) market implied volatility behaviour, we easily get that it varies as a function of $K$ and $T$.

For our comparison we consider, as a set of empirical data, the implied volatilities

$$
\Sigma_{i}=\Sigma\left(K_{i}, T_{i} ; C_{i}^{*}\right), i=1,2, \ldots, N
$$

with respect to the market prices $C_{i}^{*}$ of the options written on Russell 2000 (RUT) Index in date $18 \cdot 05 \cdot 2015$, where $N=84$, with strike price $K$ that ranges from 1200 to 1350 , for every maturity considered, i.e. for $T \in\{3,11,31,61\}$ days. The spot price was $x=1257.52$ USD and the U.S. Government Treasury Bill annualized rate, at the same date,was $r=0.2 \%$. We use the latter value 
as the riskless rate. Then we have to calibrate both the BS and the Merton model on the aforementioned data. For the BS-model we minimize the sum of squared errors

$$
\begin{aligned}
\sigma_{B S} & =\arg \min _{\sigma \in \Psi} \sum_{i=1}^{N}\left(\Sigma_{i}-\Sigma_{i}^{B S}(\sigma)\right)^{2} \\
& =\arg \min _{\Sigma_{B S} \in \Psi} \sum_{i=1}^{N}\left(\Sigma_{i}-\Sigma^{B S}\right)^{2},
\end{aligned}
$$

where

$$
\Sigma_{i}^{B S}(\sigma) \doteq \Sigma\left(K_{i}, T_{i} ; C_{B S}(\tau, x ; \sigma)\right)=\Sigma^{B S},
$$

is the implied volatility with respect to the BS price, i.e. assuming that the BSprice represents the market price of the option, hence it is obviously constant. The resulting value is

$$
\sigma_{B S}=0.1483,
$$

which is obtained considering $I_{0}$ as the initial point, and reasonable bounds as $\Psi=[0,1]$, namely we consider the market implied volatilities to range in such an interval. The calibration for the Merton model is similar, but it concerns more parameters and consists in the following non-linear least squares problem

$$
\theta_{M}=\left(\sigma_{M}, \lambda, m, \delta\right)=\arg \min _{\theta \in \Theta} \sum_{i=1}^{N}\left(\Sigma_{i}-\Sigma_{i}^{M}(\theta)\right)^{2},
$$

where

$$
\Sigma_{i}^{M}(\theta) \doteq \Sigma\left(K_{i}, T_{i} ; C_{M}(\tau, x ; \theta)\right),
$$

is the Merton implied volatility with respect to the Merton price $C_{M}(\tau, x ; \theta)$. In this case the bounds used are $\Theta=[0,1] \times[0,10] \times[-1,1] \times[0,1]$, suggested by some initial calibrations which require rather long computational time efforts, partially diminished exploiting the approach developed in [5], and by the fact that market implied volatility ranges between 0 and 1 . The resulting parameters are

$$
\sigma_{M} \simeq 0.09144, \lambda \simeq 2.878, m \simeq-0.04942, \delta \simeq 0.05443 .
$$

Fig. 2 shows how the Merton model outperforms the BS one in fitting the volatility smile for different maturities. The latter result can be quantitatively appreciated looking at Table 1 which reports the sum of squared residuals (SSR) of both models, with respect to the corresponding maturities. The BS-model becomes acceptable only for long maturities, when the volatility surface tends to 


\begin{tabular}{clc}
\hline Maturity & $S S R^{(B S)}$ & $S S R^{(M)}$ \\
\hline 3 days & 0.0992 & 0.0027 \\
\hline 11 days & 0.0195 & 0.0010 \\
\hline 31 days & 0.0091 & 0.0002 \\
\hline 61 days & 0.0079 & 0.0006 \\
\hline
\end{tabular}

Table 1: SSR of the two models for different maturities in comparison.

become flat, but it is clearly inadeguate for medium to short ones. On the other hand, even if the Merton model does not perfectly fit the smile, nevertheless the addition of the jump parameters allows to see a general big improvement if compared to the BS-model performances. 


\section{3 days}

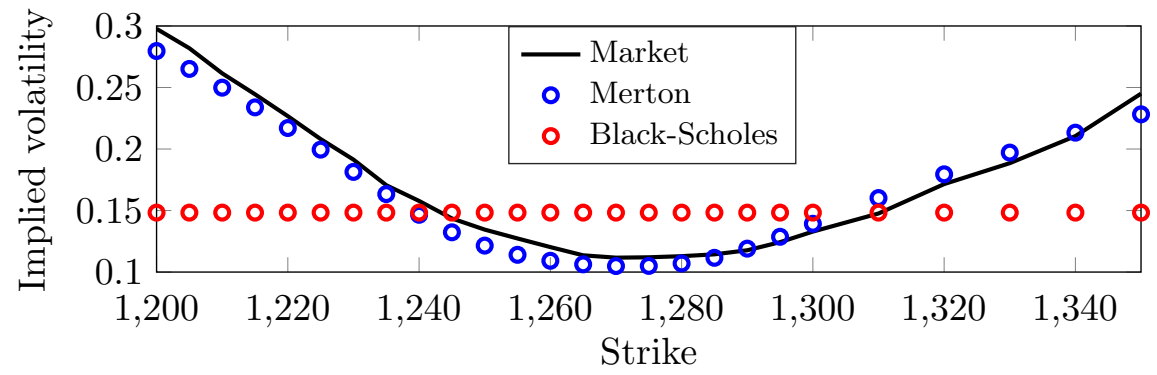

11 days

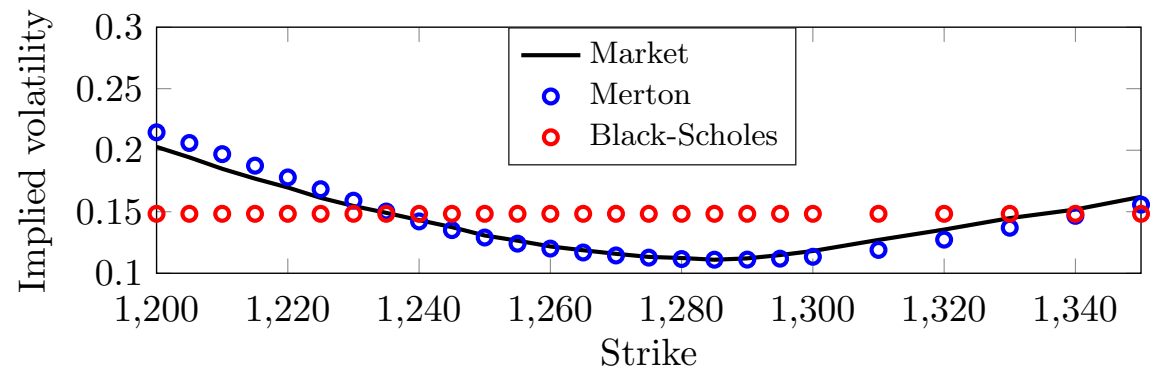

31 days

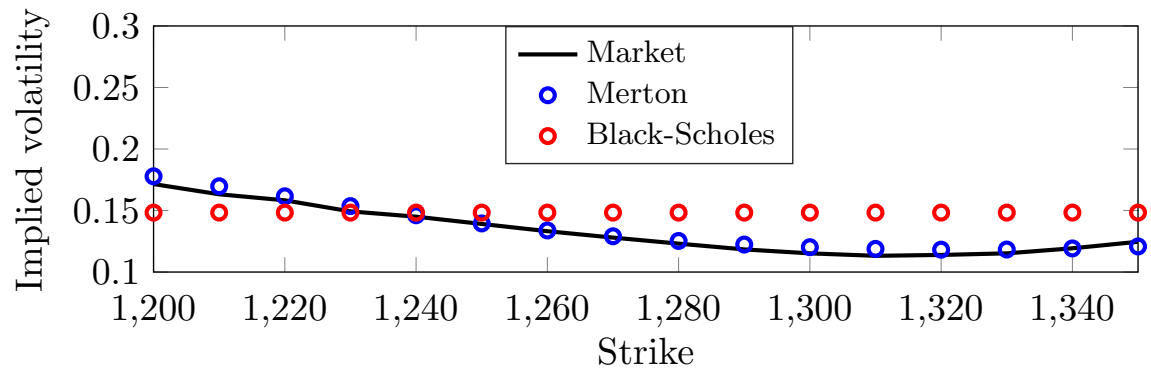

61 days

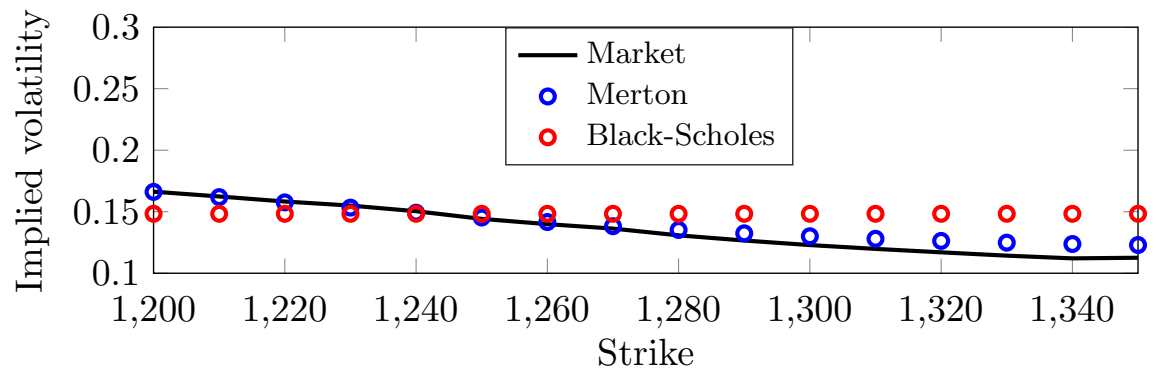

Figure 2: Simultaneous calibration of both the BS-model and the Merton model for different maturities; numerical results have been obtained following [8]. 


\section{Conclusions}

In this work we have compared the forecasting and fitness to the data performances characterizing the Black and Scholes (diffusion) model and the Merton (jump-diffusion) model, which is obtained from the BS one by adding a compensated compound Poisson process to the main stochastic differential equation. The two related market models are similar in many aspects, particularly because the financial assumptions behind them are largely the same. Nevertheless, the BS market model is complete, while the Merton one is not. The latter fact is due to the impossibility to completely hedge the risk carried by the introduction of sudden and unpredictable moves in the stock price, i.e. it is a byproduct of the continuity breaking of the underlying BS-trajectories, implied by the presence of the random (Poisson) jump component. Hence, even if one can consider the latter as an advantage carried by the BS-approach, at least in terms of mathematical simplicity and numerical tractability, the Merton model turns out to outperform the BS one, when one takes into account the performances of the two with respect to real financial data. It is worth to mention that the computational analysis needed to compare the aforementioned models require simple, but rather lengthy procedures, whose speed can be increased by truncating the related quantity of interest, namely using some asymptotic expressions of the analytical pricing formulae, as has been made, e.g., in [3, 12], and intrinsic symmetries within the treated models, as, e.g., can be made by exploiting the well known Lie analysis of related PDEs, see, e.g., [2] and references therein. In particular, moving from a theoretical comparison to an empirical one, the addition of the jump parameters results in a great improvement in option pricing and simulation of log-returns distribution. In particular the smile effect is well reproduced for short maturities, where the BS-model fails. Moreover, the log-returns leptokurtic feature is much more evident using the Merton approach instead of the BS one. In future works, we will apply an approach similar to the one proposed by Merton, namely taking random jumps into consideration, to what concerns the interest rates financial frameworks. In particular we plan to first consider the Vasicek model particularly from the point of view of its invariant measure since, see, e.g., [4], it can be uniquely determined, at least in the standard diffusive case.

\section{References}

[1] Black, F., and Scholes, M., The pricing of options and corporate liabilities, The Journal of Political Economy, 81, No. 3 (1973), 637-654, 10.1086/260062. 
[2] Cordoni, F., and Di Persio, L., Transition density for cir process by lie symmetries and application to zcb pricing (2013), International Journal of Pure and Applied Mathematics, 88 (2), pp. 239-246.

[3] Cordoni, F., and Di Persio, L., Small noise expansion for the Lévy perturbed Vasicek model (2015), International Journal of Pure and Applied Mathematics, 98 (2), pp. 291301.

[4] Cordoni, F., and Di Persio, L., Invariant measure for the Vasicek interest rate model in the Heath-Jarrow-Morton-Musiela framework (2015), Infinite Dimensional Analysis, Quantum Probability and Related Topics, 18 (3), art. no. 1550022

[5] Di Persio, L., Pellegrini, G., Bonollo, M., Polynomial chaos expansion approach to interest rate models, (2015) Journal of Probability and Statistics, No. 369053,

[6] Di Persio, L. and Frigo, M., Gibbs sampling approach to regime switching analysis of financial time series (2016), Journal of Computational and Applied Mathematics, 300, pp. 43-55.

[7] Di Persio, L. and Frigo, M., Maximum likelihood approach to markov switching models (2015), WSEAS Transactions on Business and Economics, 12, pp. 239-242.

[8] Cont, R., and Tankov, P., Financial Modelling With Jump Processes, Chapman and Hall/CRC, UK (2004).

[9] Hull, J.C, Options, Futures, And Other Derivatives, Pearson, USA (2012).

[10] Hanson, F.B., and Zhu, Z., Comparison of market parameters for jump-diffusion distributions using multinomial maximum likelihood estimation, 43rd IEEE Conference on Decision and Control, 4, 2004, 3919-3924, 10.1109/CDC.2004.1429353.

[11] Kim, C.J., Nelson, C.R., State-space Models with Regime Switching: Classical and Gibbs-sampling Approaches with Applications (1999), MIT Press, Cambridge.

[12] Marinelli, C., Di Persio, L. and Ziglio, G., Approximation and convergence of solutions to semilinear stochastic evolution equations with jumps (2013), Journal of Functional Analysis, 264 (12), pp. 2784-2816.

[13] Merton, R.C., Option pricing when underlying stock returns are discontinuous, Journal of Financial Economics, 3 (1976), 125-144.

[14] Shreve, S.E., Stochastic Calculus for Finance II: Continuous-Time Models, Springer, USA (2004).

[15] Historical Quotes for Standard and Poors 500 Index and Nasdaq 100 Index, Option Chain for Russell 2000 Index. Data taken from https://finance.yahoo.com, 2005-2015.

[16] Karatzas, I., and Shreve, S.E., Brownian Motion and Stochastic Calculus, SpringerVerlag, 1988.

[17] Rogers, L.C.G., and Williams, D., Diffusions, Markov Processes and Martingales, Cambridge Mathamatical Library, 2000. 